\title{
Effect of the quintessential dark energy on weak deflection angle by Kerr-Newmann Black
} hole

\author{
Wajiha Javed, ${ }^{1, *}$ Jameela Abbas, ${ }^{1,+}$ and Ali Övgün $2,3, \ddagger$ \\ ${ }^{1}$ Department of Mathematics, University of Education, \\ Township, Lahore-54590, Pakistan. \\ ${ }^{2}$ Instituto de Física, Pontificia Universidad Católica de Valparaíso, Casilla 4950, Valparaíso, Chile \\ ${ }^{3}$ Physics Department, Faculty of Arts and Sciences, Eastern Mediterranean University, \\ Famagusta, 99628 North Cyprus, via Mersin 10, Turkey
}

(Dated: June 12, 2019)

\begin{abstract}
In this work, we study the weak gravitational lensing in the background of Kerr-Newman black hole with quintessential dark energy. Initially, we compute the deflection angle of light by charged black hole with quintessential dark energy by utilizing the Gauss-Bonnet theorem. Firstly, we suppose the light rays on the equatorial plane in the axisymmetric spacetime. In doing so, we first find the corresponding optical metrics and then calculate the Gaussian optical curvature to utilize in GaussBonnet theorem. Consequently, we calculate the deflection angle of light for rotating charged black hole with quintessence. Additionally, we also find the deflection angle of light for Kerr-Newman black hole with quintessential dark energy. In order to verify our results, we derive deflection angle by using null geodesic equations which reduces to the deflection angle of Kerr solution with the reduction of specific parameters. Furthermore, we analyze the graphical behavior of deflection angle $\Theta$ w.r.t to quintessence parameter $\alpha$, impact parameter $b, \mathrm{BH}$ charge $Q$ and rotation parameter $a$. Our graphical analysis retrieve various results regarding to the deflection angle by the Kerr-Newman black hole with quintessential dark energy.
\end{abstract}

PACS numbers: 04.70.Dy; 04.70.Bw; 11.25.-w

Keywords: Deflection of light; rotating black hole; Deflection angle; Gauss-Bonnet theorem; Gravitational lensing, Finsler geometry, Null geodesic

\section{INTRODUCTION}

Einstein general theory of relativity is based on numerous experimental and observational tests [1]. These tests revealed that the experimental data is compatible with theoretical predictions of this theory, i.e., some of the astrophysical observations [2] like gravitational waves, gravitational lensing, black holes (BHs), wormholes etc. Einstein assumed a universe shape as a single fabric sheet, hewn from space and time. According to this theory, the force of gravity is the result of curvature in space-time and gravitational waves are ripples in the fabric of a universe that are produced by the collision of the massive objects such as BHs. Gravity bends light such phenomenon is known as gravitational lensing. The gravitational lensing is as the distribution of matter between a source and observer, which is capable of bending of light from the source to the observer and the bending of light is one of the prediction of Einstein's theory. Gravitational lensing particularly plays a vital role in the study of distribution of dark matter in universe and for imaging the most distant galaxies. The prediction that light bends when passing near massive objects is valid in Newtonian physics but is more significant in general theory of relativity. The gravitational lensing is very helpful for the detection of the BHs in the universe and this phenomenon indicates that light rays get deflected due to the curvature of spacetime. The gravitational lensing has been classified in the literature as a strong lensing and weak lensing. Strong lensing helps us to find the magnification, position and time delays of the images by BHs. Strong lensing phenomena required in many cases which yields more information from experimental frame of reference to analyze other exotic objects such that monopoles [3], boson stars [4,5], fermion stars [6, 7] etc. While the weak gravitational lensing provide a way to find the mass of astronomical objects without requiring about their composition or dynamical states. Weak lensing also investigates the cause of the accelerated expansion of the universe and also distinguish between modified gravity and dark energy. Recently, scientists detected the event horizon of BH by the reference of Event Horizon Telescope (EHT) [8]; that's why this concept has claimed a great attention

\footnotetext{
*Electronic address: wajiha.javed@ue.edu.pk

${ }^{\dagger}$ Electronic address: jameelaabbas30@gmail.com

‡Electronic address: ali.ovgun@pucv.cl; https : / / www. aovgun.com
} 
and many astronomer concentrate on it to acquire exact results [9-19].

The intriguing form of energy with positive energy density and large negative pressure is represented as dark energy (DE) [20]-[22]. There are two different approaches to deal with DE such as cosmological constant and quintessence parameter. Cosmological constant (denoted as Greek word $\Lambda$ ) describes the energy density of space [23] whereas the quintessence is a dynamical form of DE which is generated by scalar field [20]. According to some physicists, it has been proposed as a fifth fundamental force. Quintessence differs from cosmological constant as it changes over time due to that it is dynamic, otherwise the cosmological constant by definition does not change [24]. Moreover, $\omega$ is an equation of state parameter which divides DE phase in different eras such that DE phase consists of quintessence era for $-1<\omega \leq \frac{-1}{3}$ whereas $\omega=-1$ analogous to cosmological constant.

In doing so, Gibbon and Werner [25] presented a method to derive deflection angle of light rays by using Gauss Bonnet theorem (GBT). This method is use to find the integral in a finite domain bounded by the light ray. Then, Werner has enlarged this formalism and derive the angle of deflection by a Kerr-Newman BH by using the Nazim's method with Rander-Finsler metric [26]. Recently, Ishihara et al. [27-30] computed the deflection angle in a static, spherically symmetric and asymptotically flat space by using the finite distance from an observer to a light source. Moreover, the GBT has been stretched out to the wormhole geometries and non-asymptotically flat spacetime with topological effects [31-33]. A very important contribution has been currently made by Jusufi and Övgün [34] who discussed about the quantum correction effects on the deflection of light by quantum improved Kerr BH pierced in cosmic string. Stunningly, this method was shown in a suitable way to calculate the deflection angle in spacetimes with topological defects by cosmic strings and global monopoles [35-38]. This method has been utilized in various papers for different types of spacetimes [39-51].

$\mathrm{Xu}$ and Wang [52] examined the Kerr-Newman BH in the background of quintessential field. It is considerable that the Newman-Janis algorithm does not involve cosmological constant, therefore they enlarge the Kerr- Newman solution to the Kerr-Newman-AdS solution with quintessential dark energy through direct computations which satisfy the Einstein Maxwell field equations in quintessential dark energy with negative cosmological constant. Moreover, they also investigated the singularity of Kerr-Newman-AdS BH by quintessence dark energy.

In this continuation, we are going to extend this formalism for charged BH in quintessential dark energy and also for Kerr-Newman BH with quintessence. In our analysis, we focus on a non-singular domain outside of the light ray. As for asymptotically flat space, deflection angle $\Theta$ can be computed as follows [25]:

$$
\Theta=-\iint_{D_{\infty}} \mathcal{K} d \tilde{\sigma}
$$

where $\mathcal{K}$ represents the Gaussian optical curvature and $d \tilde{\sigma}$ stands for surface element of optical geometry. It is remarkable that the above expression of deflection angle just fulfill the asymptotically flat space, while for nonasymptotically flat metric only a finite distance corrections can be considered. We analyze the graphical behavior of deflection angle with respect to $\mathrm{BH}$ charge as well as impact, quintessence and rotation parameters. This paper is organized as follows: In section 2, we discuss the deflection angle of rotating charged $\mathrm{BH}$ with quintessence. In section 3, we review some basic concepts about Rander-Finsler geometry and compute the Gaussian optical curvature for deflection angle of Kerr-Newman BH with quintessence. In section 4, we calculate the deflection angle for Kerr-Newman BH with quintessence by using GBT. In section 5, we discuss the geodesic equations with the background geometry of Kerr-Newman $\mathrm{BH}$ involving quintessential dark energy and compute deflection angle. Section $\mathbf{6}$ is based on graphical analysis, we discuss deflection angle w.r.t various physical parameters. The last section comprises of concluding remarks and results obtained from graphical analysis.

\section{CHARGED BLACK HOLE WITH QUINTESSENTIAL DARK ENERGY}

The spacetime metric of charged black hole with quintessential dark energy is [54, 55]

$$
d s^{2}=-F(r) d t^{2}+F(r)^{-1} d r^{2}+r^{2} d \theta^{2}+r^{2} \sin ^{2} \theta d \phi^{2}
$$

and then we set the metric into equilateral plane with $\theta=\frac{\pi}{2}$, thus the corresponding expressions are

$$
d s^{2}=-[F(r)] d t^{2}+F(r)^{-1} d r^{2}+r^{2} d \phi^{2}
$$

where

$$
F(r)=1-\frac{2 M}{r}+\frac{Q^{2}}{r^{2}}-\frac{\alpha r^{1-3 \omega}}{r^{2}}
$$


and the optical metric is

$$
d t^{2}=\frac{1}{F(r) G(r)} d r^{2}+\frac{r^{2}}{F(r)} d \phi^{2}
$$

where $G(r)=\left[F(r)+\frac{4 m}{r} \frac{d \phi}{d t}\right]$.

Then we calculate the Gaussian curvature of the optical charged black hole as follows:

$$
\mathcal{K}=\frac{R_{\text {icciScalar }}}{2}
$$

after simplification the Gaussian optical curvature in weak field limit is stated as

$$
\begin{aligned}
\mathcal{K} & \approx-\frac{2 M}{r^{3}}+\frac{3 Q^{2}}{r^{4}}-\frac{6 M Q^{2}}{r^{5}}+\frac{1}{2 r^{6}}\left[-\left(9 \omega^{2}+9 \omega+2\right) r^{3-3 \omega}\right. \\
& \left.+18 M\left(\omega^{2}+\frac{2}{3} \omega+\frac{1}{3}\right) r^{2-3 \omega}-9 Q^{2}\left(\omega^{2}+\frac{1}{3} \omega+\frac{2}{3}\right) r^{1-3 \omega}\right] \alpha .
\end{aligned}
$$

Now, by using the GBT and Eq. (2.6), the deflection angle of charged BH with quintessential dark energy is obtained as

$$
\Theta \approx \frac{4 M}{b}+\frac{3 \pi Q^{2}}{4 b^{2}}+\frac{2 \alpha}{b}-\frac{3 \alpha \omega}{b}+\frac{6 \alpha \omega}{b}(\ln (2)-\ln (b))+\mathcal{O}\left(\alpha, M, Q^{2}\right)
$$

It is to be noted that the mass term decreases the deflection angle while quintessence parameter $\alpha$ increases the deflection angle. On the other hand, the deflection angle has an indirect relation with impact parameter $b$.

\section{GRAPHICAL ANALYSIS}

This section is devoted to discuss the graphically behavior of deflection angle $\Theta$. We also demonstrate the physical significance of these graphs to analyze the impact of $\mathrm{BH}$ charge $Q$ and quintessence $\alpha$ on deflection angle by examining the stability and instability of $\mathrm{BH}$.

\section{A. Deflection angle with Impact parameter $b$}

This subsection is based on the analysis of deflection angle $\Theta$ with impact parameter $b$ for different values of $\mathrm{BH}$ charge $Q$, and quintessence parameter $\alpha$ for fixed $M=1$ and $\omega=\frac{-2}{3}$.

(i) $Q=0.50$

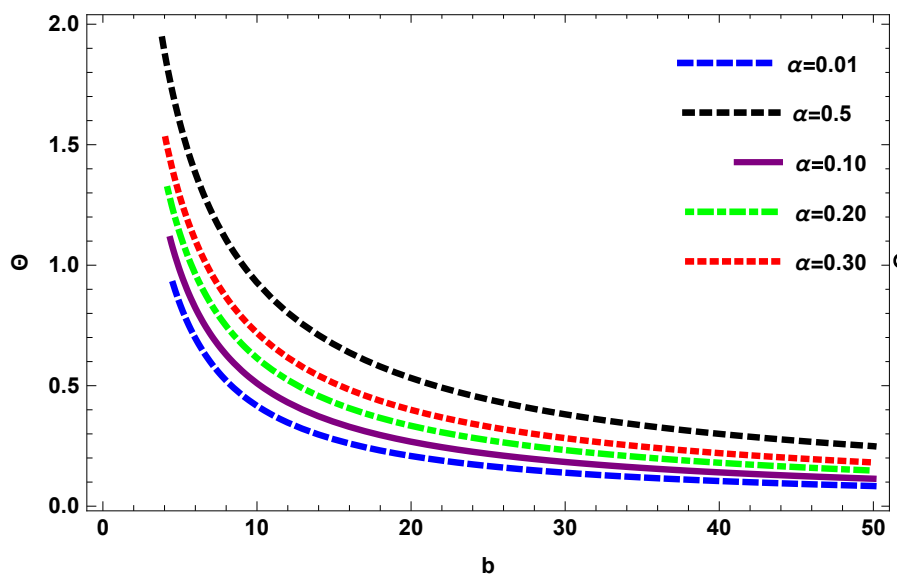

(i) $\alpha=0.20$

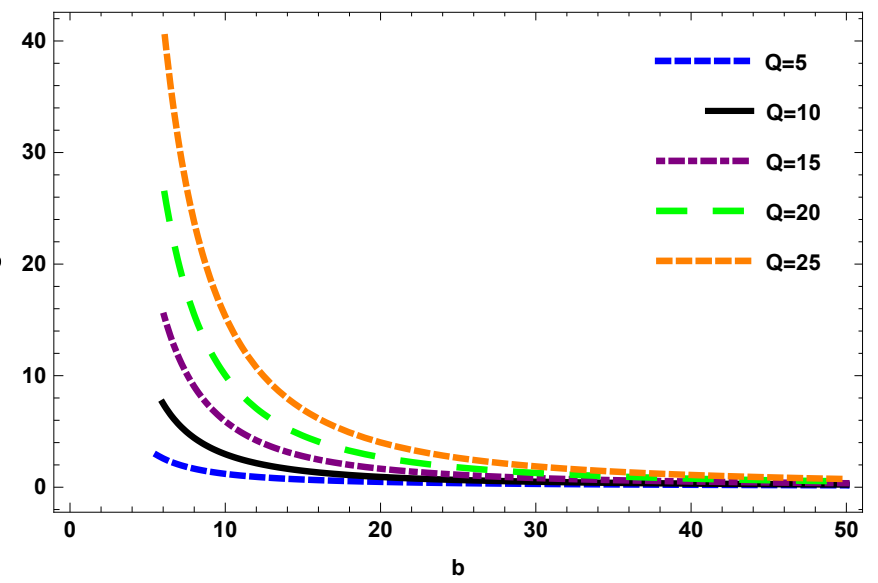

Figure 1: Relation between $\Theta$ and $b$.

- Figure 1 shows the behavior of $\Theta$ w.r.t impact parameter $b$. 
1. In figure (i), This plot shows the behavior of $\Theta$ with $b$ for fixed $Q$ and varying $\alpha$. We analyzed that deflection angle initially exponentially decreasing and then goes to positive infinity. We also observed that for $0.01 \leq \alpha \leq 1$, we obtain the stable behavior. For negative values of $\alpha$, the deflection angle is negatively decreasing which shows the unstable state of $\mathrm{BH}$. Hence, we analyzed the stable state of $\mathrm{BH}$ only for positive $\alpha$.

2. In figure (ii), This plot represents the behavior of $\Theta$ with $b$ for fixed $\alpha$ and varying $Q$ we observed that the deflection angle is gradually decreasing and then goes to positive infinity.

\section{RANDER-FINSLER GEOMETRY: KERR-NEWMAN BLACK HOLE}

In this section, we illustrate the charged rotating Kerr-Newman BH in the background of quintessential dark energy. There exist a literature related to this $\mathrm{BH}$ recently discussed by Javed and Babar [56]. For this purpose, we consider the line-element of general Kerr-Newman-AdS BH in the back ground of quintessential dark energy, i.e., [57]

$$
\begin{aligned}
d s^{2} & =\frac{\Sigma^{2}}{\Delta_{r}} d r^{2}+\frac{\Sigma^{2}}{\Delta_{\theta}} d \theta^{2}+\frac{\Delta_{\theta} \sin ^{2} \theta}{\Sigma^{2}}\left(a \frac{d t}{\Xi}-\left(r^{2}+a^{2}\right) \frac{d \phi}{\Xi}\right)^{2} \\
& -\frac{\Delta_{r}}{\Sigma^{2}}\left(d t \Xi-a \sin ^{2} \frac{d \phi}{\Xi}\right)^{2},
\end{aligned}
$$

where

$$
\begin{aligned}
& \Delta_{r}=r^{2}-2 M r+a^{2}+Q^{2}-\frac{\Lambda}{3} r^{2}\left(r^{2}+a^{2}\right)-\alpha r^{1-3 \omega}, \\
& \Delta_{\theta}=1+\frac{\Lambda}{3} a^{2} \cos ^{2} \theta, \quad \Xi=1+\frac{\Lambda}{3} a^{2}, \quad \Sigma^{2}=r^{2}+a^{2} \cos ^{2} \theta,
\end{aligned}
$$

where $M$ is the mass, $Q$ is $\mathrm{BH}$ charge, $a$ represents spin and $\alpha$ is quintessence parameter representing dark energy. For $\Lambda=0$, this solution reduces to a charged rotating $\mathrm{BH}$ solution in the presence of quintessential dark energy. Thus, for the sake of simplicity, we consider $\Lambda=0$ and also set the metric into equilateral plane with $\theta=\frac{\pi}{2}$, thus the corresponding expressions are

$$
\begin{aligned}
& \Delta_{r}=r^{2}-2 M r+a^{2}+Q^{2}-\alpha r^{1-3 \omega}, \\
& \Delta_{\theta}=1, \quad \Xi=1, \quad \Sigma^{2}=r^{2} .
\end{aligned}
$$

It is appropriate to first find the black hole optical metric by imposing the null condition $d s^{2}=0$, and solving the space-time metric for $d t$, which yield the generic form as

$$
d t= \pm \sqrt{\xi_{i j} v^{i} v^{j}}+\gamma_{i} v^{i}
$$

moreover, the expression $\xi_{i j} v^{i} v^{j}$ can be derived as follows

$$
\xi_{i j} v^{i} v^{j}=\frac{r^{4}}{\Delta_{r}\left(\Delta_{r}-a^{2}\right)} d r^{2}+\left(\frac{\left(r^{2}+a^{2}\right)-a^{2} \Delta_{r}}{\Delta_{r}-a^{2}}+\frac{a^{2}\left(\left(r^{2}+a^{2}\right)+\Delta_{r}\right)^{2}}{\Delta_{r}-a^{2}}\right) d \phi^{2},
$$

while, $\gamma_{i} v^{i}$ can be expressed as

$$
\gamma_{i} v^{i}=-\frac{a\left(r^{2}+a^{2}-\Delta_{r}\right)}{\Delta_{r}-a^{2}} d \phi .
$$

The deformed form of the given metric in Finsler-Randers type can be expressed as [26]

$$
\begin{aligned}
\tilde{\mathcal{F}}\left(r, \phi, \frac{d r}{d t}, \frac{d \phi}{d t}\right) & =\sqrt{\frac{r^{4}}{\Delta_{r}\left(\Delta_{r}-a^{2}\right)}\left(\frac{d r}{d t}\right)^{2}+\frac{\left(r^{2}+a^{2}\right)-a^{2} \Delta_{r}}{\Delta_{r}-a^{2}}} \\
& \times \sqrt{\frac{a^{2}\left(\left(r^{2}+a^{2}\right)+\Delta_{r}\right)^{2}}{\Delta_{r}-a^{2}}\left(\frac{d \phi}{d t}\right)^{2}-\frac{a\left(r^{2}+a^{2}\right)}{\Delta_{r}-a^{2}}} \\
& +\frac{a \Delta_{r}}{\Delta_{r}-a^{2}}\left(\frac{d \phi}{d t}\right) .
\end{aligned}
$$


The Randers-Finsler optical metric $\tilde{\mathcal{F}}$ is actually use to calculate the null geodesics in our physical metric. Since $d t=\tilde{\mathcal{F}}(u, d u)$, on the other hand by Fermat's principle light rays $(\tilde{\gamma})$ are selected by the following condition

$$
\delta \int_{\tilde{\gamma}} d t=\delta \int_{\tilde{\gamma} \tilde{\mathcal{F}}} \tilde{\mathcal{F}}(u, \dot{u}) d t=0
$$

Notice that these light rays $(\tilde{\gamma})$ are also geodesic $\left(\tilde{\gamma}_{\mathcal{F}}\right)$ of the Rander metric. Thus, it is remarkable that Rander-Finsler metric $\tilde{\mathcal{F}}$ generalizes the Fermat's principle. The Rander-Finsler metric in the sense that the Hessian

$$
g_{i j}(u, v)=\frac{1}{2} \frac{\partial^{2} \tilde{\mathcal{F}}^{2}(u, v)}{\partial v^{i} \partial v^{j}}
$$

is positive definite, where $u \in \mathcal{M}, v \in T_{u} \mathcal{M}$. In order to continue along the construction of a Riemannian manifold $(\mathcal{M}, \bar{g})$ which osculate with the Randers manifold $(\mathcal{M}, \tilde{\mathcal{F}})$ by applying the so-called Nazim's method. In order to calculate the Riemannian metric $\bar{g}$ from the Hessian (2.6), we consider a non-zero smooth vector field $\bar{g}$ over $\mathcal{M}$ containing the tangent vector with the geodesic $\tilde{\gamma}_{\tilde{\mathcal{F}}}$, stated as $\bar{v}\left(\tilde{\gamma}_{\tilde{\mathcal{F}}}\right)=\dot{u}$, i.e.,

$$
\tilde{g_{i j}}(u)=g_{i j}(u, \tilde{v}(u)) \text {. }
$$

The geometrical quantities which osculate the Riemannian manifold, for illustration in $T_{u} \mathcal{M}$ angle between vectors can be measured by the metric $\bar{g}$. Thus, the geodesic $\tilde{\gamma}_{\tilde{\mathcal{F}}}$ of Randers manifold is also a geodesic $\tilde{\gamma}_{\bar{g}}$ of Riemanian manifold, i.e., $\left(\tilde{\gamma} \tilde{\mathcal{F}}=\tilde{\gamma}_{\bar{g}}\right)$ by supposition

$$
\ddot{u}^{i}+\Gamma_{j k}^{i}(u, \dot{u}) \dot{u}^{j} \dot{u}^{k}=\ddot{u}^{i}+\tilde{\Gamma}_{j k}^{i}(u) \dot{u}^{j} \dot{u}^{k}=0,
$$

to start with the Randers-Optical geometry $(\mathcal{M}, \tilde{\mathcal{F}})$, suppose in the equatorial plane, a region $D_{R} \subset \mathcal{M}$ which is bounded by the light ray $\tilde{\gamma}_{\tilde{\mathcal{F}}}$ with co-ordinate radius $b$ and a circular curve $\tilde{\gamma}_{R}$ with co-ordinate radius $R$. These curves are as follows [26]

$$
\begin{gathered}
\tilde{\gamma}_{\tilde{\mathcal{F}}}: u^{i}(x)=\mu^{i}(x), x \in[0, n] \\
\tilde{\gamma}_{\tilde{\mathcal{R}}}: u^{i}(x)=\xi^{i}(x), x \in\left[0, n^{\star}\right] .
\end{gathered}
$$

Now, consider $\zeta=x / n \in(0,1)$ across the curve $\tilde{\gamma}_{\tilde{\mathcal{F}}}$ and $\zeta^{\star}=1-x / n^{\star} \in(0,1)$ across the $\tilde{\gamma}_{\tilde{\mathcal{R}}}$, each point on the geodesic $\tilde{\gamma}_{\tilde{F}}$ directly paired with one point $\xi^{i}\left(n^{\star}\right)$ on curve $\tilde{\gamma}_{\tilde{\mathcal{R}}}$ by letting $\varsigma=\varsigma^{\star}$. On the other hand, we also analyze that there is a family of smooth curves $u^{i}(\tilde{\sigma}, \varsigma)$, in such a way that for each point pair there is precisely one curve which touches the boundary of $\tilde{\gamma}_{\mathcal{F}}$ and $\tilde{\gamma}_{\tilde{R}}$. In other words we can say that when $\mu^{i}(\varsigma)=u^{i}(0, \varsigma)$ then $u^{i}(\tilde{\sigma}, \varsigma)$ touches the boundary of $\tilde{\gamma}_{\tilde{\mathcal{F}}}, \tilde{\sigma}$ represent as a new parameter. Then following equation holds

$$
\dot{\mu}^{i}(\varsigma)=\frac{d \mu^{i}}{d x}(\varsigma)=\frac{d u^{i}}{d \tilde{\sigma}}(0, \varsigma)
$$

Similarly, we can say that when $\xi^{i}(\tilde{\sigma}, \varsigma)=u^{i}(1, \varsigma)$ then $u^{i}(\tilde{\sigma}, \varsigma)$ touches the curve $\tilde{\gamma}_{\tilde{\mathcal{R}}}$ which is as follows

$$
\dot{\zeta}^{i}(\varsigma)=\frac{d \xi^{i}}{d x}(\varsigma)=\frac{d u^{i}}{d \tilde{\sigma}}(1, \varsigma)
$$

In general, we can say that a smooth and non zero tangent vector field

$$
\tilde{v}^{i}(u(\tilde{\sigma}, \varsigma))=\frac{d u^{i}}{d \tilde{\sigma}}(\tilde{\sigma}, \varsigma),
$$

with a group of smooth curves which fulfill the accompanying relation [26]

$$
\begin{aligned}
u^{i}(\tilde{\sigma}, \varsigma)=\mu^{i}(\varsigma)+\dot{\zeta}^{i}(\varsigma) \tilde{\sigma} & +\mathcal{Y}(\varsigma) \tilde{\sigma}^{2}+\mathcal{Z}(\varsigma) \tilde{\sigma}^{3} \\
& +\mathcal{A}^{i}(\tilde{\sigma}, \varsigma)(1-\tilde{\sigma})^{2} \tilde{\sigma}^{2}
\end{aligned}
$$


where

$$
\begin{gathered}
\mathcal{Y}(\varsigma)=3 \xi^{i}(\varsigma)-3 \mu^{i}(\varsigma)-\dot{\xi}^{i}(\varsigma)-2 \dot{\mu}^{i}(\varsigma) \mu^{i}(\varsigma), \\
\mathcal{Z}(\varsigma)=2 \mu^{i}(\varsigma)-2 \xi^{i}(\varsigma)+\dot{\zeta}^{i}(\varsigma)+\dot{\mu}^{i}(\varsigma) .
\end{gathered}
$$

As we know that the metric we use is asymptotically plan, so for the deflection angle of the planer light ray we choose

$$
r(\phi)=\frac{b}{\sin \phi}
$$

where $b$ gives the minimal radial distance of the light ray and is known as impact parameter. Now we make the following leading terms for the vector field

$$
\bar{v}^{r}=\frac{d r}{d x}=-\cos \phi, \bar{v}^{\phi}=\frac{d \phi}{d x}=\frac{\sin ^{2} \phi}{b} .
$$

It is well-known that the vector field which we use is determined by the light ray equation $r_{\tilde{\gamma}}$. Where our particular light ray equation shows a straight line approximation, for this straight line approximation we verify our angle of deflection.

\section{A. Gaussian Curvature}

In this section we compute the metric components. For this, we use Eqs. (2.6) and (2.17), we get the following non-zero components

$$
\begin{aligned}
\bar{g}_{r r} & =\frac{r^{4}}{\left(\alpha r^{\left.1-3 \omega-Q^{2}+2 m r-r^{2}\right)^{2}},\right.} \\
\bar{g}_{r \phi} & =2 \frac{\left(\left((\cos (\phi))^{4} r^{2}+\left(b^{2}-2 r^{2}\right)(\cos (\phi))^{2}+r^{2}\right) r\left(r^{\omega}\right)^{3}+\bar{\Phi} \alpha\right) m a b^{3}(\cos (\phi))^{3}}{\left((\cos (\phi))^{4} r^{2}+\left(b^{2}-2 r^{2}\right)(\cos (\phi))^{2}+r^{2}\right)^{5 / 2} r^{2}\left(r^{\omega}\right)^{3}}, \\
\bar{g}_{\phi \phi} & =-\frac{\left(\left(\alpha r^{1-3 \omega}-Q^{2}-r^{2}\right)(\sin (\phi))^{4}-(\cos (\phi))^{2} b^{2} m\right) r^{4}}{\left(\left(\alpha r^{1-3 \omega}-Q^{2}-r^{2}\right)(\sin (\phi))^{4}-(\cos (\phi))^{2} b^{2}\right)\left(\alpha r^{1-3 \omega}-Q^{2}-r^{2}\right)},
\end{aligned}
$$

with $\bar{\Phi}=2\left(7 / 4(\cos (\phi))^{4} r^{2}+\left(b^{2}-7 / 2 r^{2}\right)(\cos (\phi))^{2}+7 / 4 r^{2}\right)$.

By neglecting the higher order terms of the angular momentum $a$ we get the determinant of this metric as follows

$$
\operatorname{det} \bar{g}=-\frac{r^{8}\left(r^{1-3 \omega}(\sin (\phi))^{4} \alpha-(\sin (\phi))^{4} r^{2}-(\cos (\phi))^{2} b^{2} m\right)}{\left(\alpha r^{1-3 \omega}+2 m r-r^{2}\right)^{2}\left(r^{1-3 \omega}(\sin (\phi))^{4} \alpha-(\sin (\phi))^{4} r^{2}-(\cos (\phi))^{2} b^{2}\right)\left(\alpha r^{1-3 \omega}-r^{2}\right)}
$$

The Gaussian optical curvature $\mathcal{K}$, is defined as:

$$
\mathcal{K}=\frac{1}{\sqrt{\operatorname{det} \bar{g}}}\left[\frac{\partial}{\partial \phi}\left(\frac{\sqrt{\operatorname{det} \bar{g}}}{\bar{g}_{r r}} \Gamma_{r r}^{\phi}\right)-\frac{\partial}{\partial r}\left(\frac{\sqrt{\operatorname{det}_{\bar{g}}}}{\bar{g}_{r r}} \Gamma_{r \phi}^{\phi}\right)\right] .
$$

So by using Christopher symbols and the non-zero metric components, we get

$$
\begin{array}{r}
\mathcal{K} \approx-\frac{2 M}{r^{3}}+\frac{3 Q^{2}}{r^{4}}-\frac{6 M Q^{2}}{r^{5}}+\frac{1}{2 r^{6}}\left[-\left(9 \omega^{2}+9 \omega+2\right) r^{3-3 \omega}+18 M\left(\omega^{2}+\frac{2}{3} \omega+\frac{1}{3}\right) r^{2-3 \omega} 9 Q^{2}\left(\omega^{2}+\frac{1}{3} \omega+\frac{2}{3}\right) r^{1-3 \omega}\right] \alpha \\
+\frac{15 a M H(r, \phi, \alpha)}{r^{9}}(31)
\end{array}
$$

The last term gives the rotation. Where the function $H(r, \phi)$ is as follows 


$$
\begin{aligned}
H(r, \phi, \alpha) & =\left(r^{2}+a^{2}+Q^{2}-\alpha r^{1-3 \omega}\right) r^{-9}\left(r^{2} \sin ^{4} \phi+b^{2} \cos ^{2} \phi\right)^{\frac{-7}{2}} \\
& \times\left[r^{10} \sin ^{12} \phi-\frac{b^{2} r^{4} \sin ^{10} \phi}{2}\left(r^{2}+a^{2}+Q^{2}-\alpha r^{1-3 \omega}\right)^{2}\right. \\
& +\frac{\cos ^{2} \phi}{2}\left[-4 r^{4}-71 r^{2}\left(a^{2}+Q^{2}-\alpha r^{1-3 \omega}\right) \frac{49}{11}\right. \\
& \left.-10\left(a^{2}+Q^{2}-\alpha r^{1-3 \omega}\right)^{2}\right] b^{2} r^{4} \sin ^{8} \phi+4 b^{3} \cos ^{3} \phi \\
& \times\left(r^{2}+\frac{1}{2}\left(a^{2}+Q^{2}-\alpha r^{1-3 \omega}\right)\right)\left(r^{2}+a^{2}+Q^{2}-\alpha r^{1-3 \omega}\right) \\
& \times r^{3} \sin ^{7} \phi+b^{2} \cos ^{2} \phi\left(2 b^{2}-5 r^{2} \cos ^{2} \phi\right)\left(r^{2}+a^{2}\right. \\
& \left.+Q^{2}-\alpha r^{1-3 \omega}\right)^{2} r^{2} \sin ^{6} \phi+8 b^{3} \cos ^{4} \phi\left(r^{2}+\frac{1}{2}\left(a^{2}+Q^{2}\right.\right. \\
& \left.\left.-\alpha r^{1-3 \omega}\right)\right)\left(r^{2}+a^{2}+Q^{2}-\alpha r^{1-3 \omega}\right) r^{3} \sin ^{5} \phi-3 b^{6} \\
& \times \cos ^{6} \phi\left(r^{2}-\frac{1}{3}\left(-a^{2}-Q^{2}-\alpha r^{1-3 \omega}\right)\right)+\cos ^{4} \phi\left(\frac{-9}{2} r^{2}\right. \\
& \left.+\frac{8}{3} r^{2}-\frac{7}{2}\right) b^{2} r^{2} \sin ^{4} \phi+5 b^{4} \cos ^{6} \phi\left(r^{2}+a^{2}+Q^{2}\right. \\
& \left.-\alpha r^{1-3 \omega}\right) r^{2} \sin ^{2} \phi-2 b^{5} r^{3} \cos ^{6} \phi\left(r^{2}+4\left(a^{2}+Q^{2}\right.\right. \\
& \left.\left.-\alpha r^{1-3 \omega}\right)\right) \sin ^{2}-r^{3} b^{5} \cos ^{4} \phi \sin ^{3} \phi\left(\left(r^{2}+4\left(a^{2}+Q^{2}\right.\right.\right. \\
& \left.\left.\left.-\alpha r^{1-3 \omega}\right)\right)\right] .
\end{aligned}
$$

Where the Gaussian optical curvature depends on the BH parameters, $a, M$,

$Q^{2}, \alpha$. In the next part, by using Gaussian optical curvature we can compute angle of deflection.

\section{DEFLECTION ANGLE USING GAUSS BONNET THEOREM}

Let the domain $\left(D_{R}, \bar{g}\right)$ be a simply connected over the osculating Riemannian manifold $(\mathcal{M}, \bar{g})$ along the boundaries of $\tilde{\gamma}_{\bar{g}}$ and $\tilde{\gamma}_{\tilde{R}}$. Furthermore, we define Gaussian curvature as a $\mathcal{K}$ of $(\mathcal{M}, \bar{g})$ and geodesic curvature represents as $\kappa$ of $\partial D_{\tilde{R}}=\tilde{\gamma}_{\bar{\delta}} \cup \tilde{\gamma}_{\tilde{R}}$. Thus by the Gauss-Bonnet theorem

$$
\iint_{D_{R}} K d \tilde{\sigma}+\oint_{\partial D_{R}} \kappa d x+\sum_{n} \Theta^{n}=2 \pi \mathcal{X}\left(D_{R}\right) .
$$

Here $d \tilde{\sigma}$ is defined as the surface element, $\Theta_{n}$ is the exterior angle at the $n t h$ vertex, $\mathcal{X}\left(D_{R}\right)$ be defined as Euler characteristic number. The geodesic curvature use to determine the deviation from geodesic. Since in case of geodesics $\tilde{\gamma}_{\bar{g}}$ the geodesic curvature vanishes i-e $\kappa\left(\tilde{\gamma}_{\bar{g}}\right)=0$, if an only if, $\tilde{\gamma}$ is geodesic. Now, our main focuss on calculating $\mathcal{\kappa}\left(\tilde{\gamma}_{\bar{\delta}}\right)$. Which is defined as

$$
\kappa\left(\tilde{\gamma}_{R}\right)=\left|\nabla_{\dot{\tilde{\gamma}} R} \dot{\tilde{\gamma}} \mathcal{R}\right| .
$$

Now we consider $\tilde{\gamma}_{R}:=r(\phi)=R=$ const, in such a way the radial part states that

$$
\left(\nabla_{\dot{\tilde{\gamma}} R} \dot{\tilde{\gamma}} \mathcal{R}\right)^{r}=\dot{\tilde{\gamma}}_{R}^{\phi}\left(\partial_{\phi} \dot{\tilde{\gamma}}_{R}^{r}\right)+\bar{\Gamma}_{\phi \phi}^{r}\left(\dot{\tilde{\gamma}}_{R}^{\phi}\right)^{2}
$$

It is obvious that the first term vanishes, where by using the unit speed condition $\left(\bar{g}_{\phi \phi} \dot{\hat{\gamma}}_{R}^{\phi} \dot{\tilde{\gamma}}_{R}^{\phi}\right)=1$ we find the second term. Seeing that our optical geometry is asymptotically Euclidean for geodesic curvature, At $R \rightarrow \infty$ the geodesic curvature reduces to $\kappa\left(\tilde{\gamma}_{R}\right) \rightarrow \frac{1}{R}$. If we consider $R \rightarrow \infty$ the jump angles $\left(\Theta_{O}, \Theta_{D}\right)$ become $\pi$, in other words we say that the sum of jump angles to the source of light $D$ and the position of observer $O$ satisfies $\Theta_{O}+\Theta_{D} \rightarrow \pi$. Now, for 
constant $R$ the optical metric yields

$$
\begin{aligned}
\lim _{R \rightarrow \infty} d x & =\lim _{R \rightarrow \infty}\left[\sqrt{\frac{r^{4}}{\Delta_{r}\left(\Delta_{r}-a^{2}\right)}\left(\frac{d r}{d t}\right)^{2}}\right. \\
& \times \sqrt{\frac{\left(r^{2}+a^{2}\right)^{2}-a^{2} \Delta_{r}}{\Delta_{r}-a^{2}}+\frac{a^{2}\left(\left(r^{2}+a^{2}\right)+\Delta_{r}\right)^{2}}{\Delta_{r}-a^{2}}\left(\frac{d \phi}{d t}\right)^{2}} \\
& \left.-a\left(\frac{\left(r^{2}+a^{2}\right)+\Delta_{r}}{\Delta_{r}-a^{2}}\right)\right] d \phi \\
& \rightarrow R d \phi,
\end{aligned}
$$

On the other hand we use

$$
\lim _{R \rightarrow \infty} h(R) \rightarrow 1
$$

Hence, we represent that

$$
\lim _{R \rightarrow \infty} \kappa\left(\tilde{\gamma}_{R}\right) \frac{d x}{d \phi} \rightarrow 1
$$

By supposition, the source and observer are in the asymptotically Euclidean region, consequently the last equation plainly shows our conclusion that our optical metric is asymptotically Euclidean. So by Gauss-Bonnet theorem geodesic curvature yields that

$$
\iint_{D_{R}} \mathcal{K} d D+\oint_{\tilde{\gamma}_{R}} \kappa d x={ }^{R \rightarrow \infty} \iint_{D_{R}} \mathcal{K} d \tilde{\sigma}+\int_{0}^{\pi+\hat{\alpha}} d \phi=\pi
$$

hence, we obtain

$$
\Theta=-\iint_{D_{\infty}} \mathcal{K} d \tilde{\sigma}
$$

Now by using the straight line approximation of the light ray $\left(r=\frac{b}{\sin \phi}\right)$, we get the total deflection angle

$$
\Theta \simeq \frac{4 M}{b}+\frac{2 \alpha}{b}-\frac{3 \alpha \omega}{b}-\frac{3 Q^{2}}{4 b^{2}} \pm \frac{4 M a}{b^{2}}+\mathcal{O}(M),
$$

where the positive and negative signs denoted the reversed and rotational movement of light rays.

\section{NULL GEODESICS}

Now, by using the variational principle $\delta \int \mathcal{L} d D=0$ [53], we find the deflection angle. For this we set $\theta=\frac{\pi}{2}$ and $\dot{\theta}=0$, then we find the following equation for lagrangian

$$
\begin{aligned}
2 \mathcal{L} & =-\left(\frac{\Delta_{r}-a^{2}}{\Sigma^{2} \Xi^{2}}\right) \dot{t}^{2}+\left(\frac{\left(r^{2}+a^{2}\right)^{2}-a^{2} \Delta_{r}}{\Sigma^{2} \Xi^{2}}\right) \dot{\phi}^{2}+\frac{\Sigma^{2}}{\Delta_{r}} \dot{r}^{2} \\
& -\left(\frac{2 a\left(\left(r^{2}+a^{2}\right)-\Delta_{r}\right)}{\Sigma^{2} \Xi^{2}}\right) \dot{\phi} \dot{t}
\end{aligned}
$$

furthermore $\dot{p}=\frac{d p}{d \tau}$ and $\tau$ stands for affine parameter along the geodesic. Since the metric coefficients do not depend on cyclic coordinates $(t, \phi)$, then the conjugate momenta of these cyclic coordinates are represented by $\left(\Omega_{t}, \Omega_{\phi}\right)$ and are preserved. Then the equations of motion are derived from $\dot{\Omega}_{p}-\frac{\partial \mathcal{L}}{\partial p}=0$ leading to

$$
\dot{\Omega}_{t}=0, \dot{\Omega}_{\phi}=0
$$


here $\Omega_{p}=\frac{\partial \mathcal{L}}{\partial \dot{p}}$ are conjugate momenta to the space-time coordinate $p$, and for lagrangian represented by

$$
\begin{aligned}
\Omega_{t} & =-\frac{\Delta_{r}}{r^{2} \Xi^{2}} \dot{t}-a \frac{\left(r^{2}+a^{2}-\Delta_{r}\right)}{r^{2} \Xi^{2}} \dot{\phi} \equiv-F, \\
\Omega_{r} & =\frac{r^{2}}{\Delta_{r}} \dot{r}^{2} \\
\Omega_{\phi} & =-a \frac{\left(r^{2}+a^{2}-\Delta_{r}\right)}{r^{2} \Xi^{2}} \dot{t}+\frac{r^{2}+a^{2}}{\Xi^{2}} \dot{\phi} \equiv N,
\end{aligned}
$$

where $F$ and $N$ are constants of motion for a test particle, relating to the energy at infinity and the angular momentum, respectively. Thus the Hamiltonian for a test particle is given by

$$
\begin{gathered}
\mathcal{H}=\Omega_{t} \dot{t}+\Omega_{\phi} \dot{\phi}+\Omega_{r} \dot{r}-\mathcal{L}, \\
2 \mathcal{H}=-F \dot{t}+N \dot{\phi}+\frac{r^{2}}{\Delta_{r}} \dot{r}^{2} \equiv-M^{2} .
\end{gathered}
$$

Now, we consider only massless particles and taking the condition $M^{2}=0$ for photons. Then we get the following equations of motion

$$
\begin{aligned}
\dot{t} & =\left(-F r^{2}+a N\left(1-\frac{1}{r^{2}}\left(a^{2}+Q^{2}\right)+\alpha r^{-1-3 \omega}\right)\right)\left(r^{2}+a^{2}\right. \\
& \left.+Q^{2}-\alpha r^{1-3 \omega}\right)^{-1} \\
\dot{\phi} & =\left(N+a F\left(a^{2}+Q^{2}-\alpha r^{1-3 \omega}\right)\right)\left(r^{2}+a^{2}+Q^{2}-\alpha r^{1-3 \omega}\right)^{-1} \\
\dot{r}^{2} & =\left(-r^{2} F^{2}+a F N\left(1-\frac{1}{r^{2}}\left(a^{2}+Q^{2}\right)+\alpha r^{-1-3 \omega}\right)\right. \\
& \left.-r^{2} N(N+a F)\right)\left(1+\frac{1}{r^{2}}\left(a^{2}+Q^{2}\right)-\alpha r^{-1-3 \omega}\right)^{-2} .
\end{aligned}
$$

The closest distance $r_{0}$ for the metric (8) can be calculated by taking $\dot{r}=0$, such that

$$
\frac{d \phi}{d r}=\frac{r_{0}}{b}=\sqrt{1-\left(\frac{a}{b}\right)^{2}+r_{0}^{2}\left(1-\frac{a}{b}\right)^{2}\left(R^{2}-2 M\right)},
$$

where

$$
R^{2}=a^{2}+Q^{2}-\alpha r^{1-3 \omega}
$$

Then, for bending angle

$$
\Theta=2 \int_{r_{0}}^{\infty}\left|\frac{d \phi}{d r}\right| d r-\pi
$$

after simplification we get

$$
\Theta \simeq \frac{4 M}{b}+\frac{2 \alpha}{b}-\frac{3 \alpha \omega}{b}-\frac{3 Q^{2}}{4 b^{2}} \pm \frac{4 M a}{b^{2}}+\mathcal{O}(M)
$$

now we utilize the change of variables such as $w=r_{0} / r$; Moreover, we replace the impact parameter stated as Eq. (2.47), then we extend in Taylor series about $Q, M, a, \alpha$ and $\omega$. Hence we suppose $r_{0} \simeq b$.

\section{GRAPHICAL ANALYSIS}

This section is devoted to discuss the graphical behavior of deflection angle $\Theta$. We also demonstrate the impact of $\mathrm{BH}$ charge $Q$, quintessence $\alpha$, rotation $a$ and impact $b$ parameters on deflection angle by examining the stability and instability of $\mathrm{BH}$. 


\section{A. Deflection angle with Impact parameter $b$}

This subsection is based on the analysis of deflection angle $\Theta$ with impact parameter $b$ for different values of rotation parameter $a, \mathrm{BH}$ charge $Q$, and quintessence parameter $\alpha$ for fixed $M=1$ and $\omega=\frac{-2}{3}$.
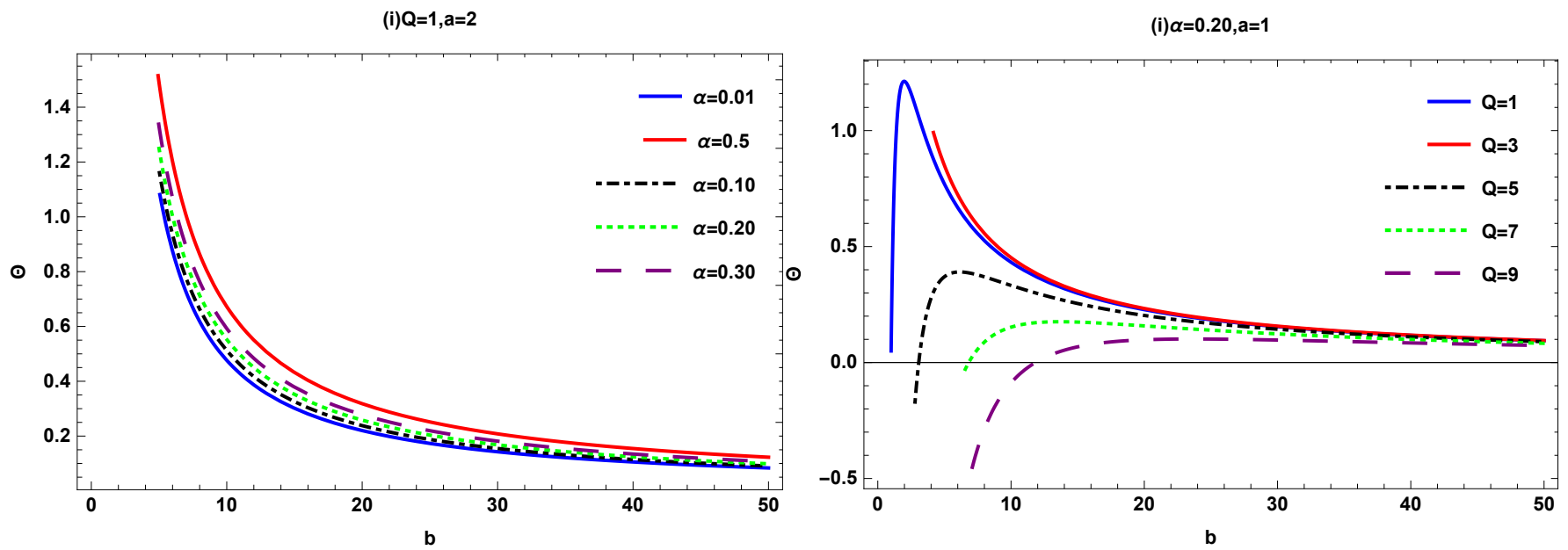

Figure 2: Relation between $\Theta$ and $b$.

- Figure 2 shows the behavior of $\Theta$ w.r.t impact parameter $b$.

1. In figure (i), This plot shows the behavior of $\Theta$ with $b$ for fixed $Q, a$ and varying $\alpha$. We analyzed that deflection angle gradually decreasing and then goes to positive infinity. We analyzed the stable behavior of deflection angle only for positive values of quintessence parameter.

2. In figure (ii), This plot represents the behavior of $\Theta$ with $b$ for fixed $\alpha, a$ and varying $Q$ we observed that the deflection angle initially increasing from negative to positive and then gradually decreasing which shows the stable behavior.

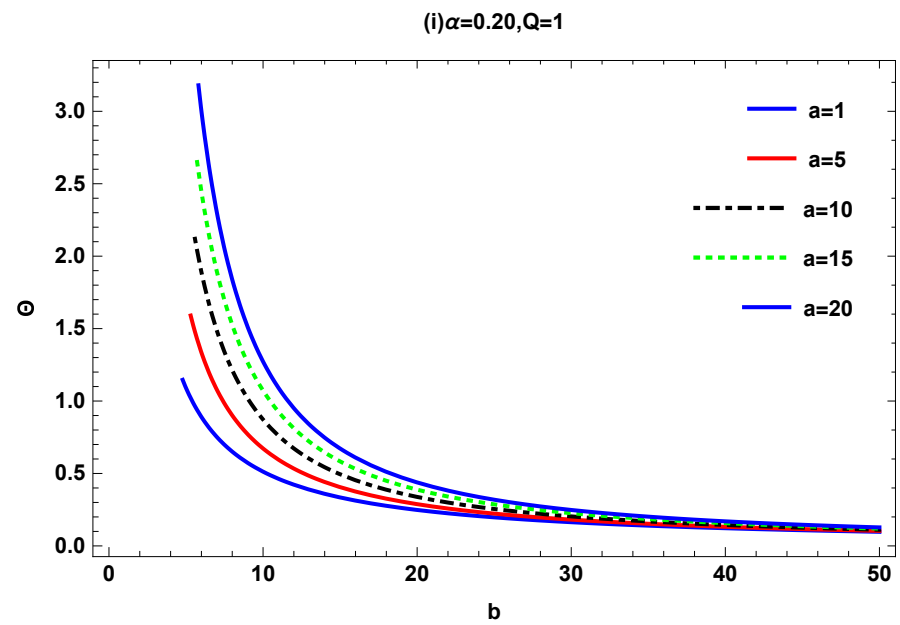

Figure 3: Relation between $\Theta$ and $b$.

- Figure 3 shows the behavior of $\Theta$ w.r.t impact parameter $b$.

This figure shows the behavior of $\Theta$ with $b$ for fixed $Q, \alpha$ and varying $a$. We observed that for negative value of $a$ the deflection angle is negatively decreasing but for $a>0$ deflection angle initially exponentially decreasing and then goes to positive infinity.

\section{CONCLUSION}

In this paper, we have analyzed the weak gravitational lensing in the background of Kerr-Newman BH with quintessential dark energy. Initially, we have found the deflection angle of light by slowly rotating charged $\mathrm{BH}$ 
with quintessence by means of GBT. For this purpose, firstly we have consider the photon rays into equatorial plane. Afterthat we have obtained the corresponding optical metric and calculated the Gaussian optical curvature in leading order terms. Consequently, we have calculated the deflection angle of light by slowly rotating charged $\mathrm{BH}$ with quintessence. We also investigated the graphical behavior of impact parameter on deflection angle.

Deflection angle by slowly rotating Charged $\mathrm{BH}$ with Quintessence with respect to Impact parameter:

- The behavior of $\Theta$ w.r.t $b$ can be analyzed.

- For particular value of $b$, we can only obtain the stable behavior for $0.01 \leq \alpha \leq 1$.

- We observe that for the variation of $Q$ the deflection angle gradually decreasing, which indicates that the $\mathrm{BH}$ evolution appears initially from physical stable state to unstable.

In addition, we have also obtained the deflection angle of light by Kerr-Newman BH. Moreover, for verification of our results we have used the null geodesics method and calculated the deflection angle of light by Kerr-Newman $\mathrm{BH}$ with quintessence. Our proposed deflection angle of light by Kerr-Newman BH with quintessence reduced into Schwarzschild $\mathrm{BH}$ solution by the reduction of $\mathrm{BH}$ charge and if we take $\alpha=Q=0$ then our results reduced into Kerr solution. The Kerr-Newman BH solution may be applicable in astrophysics. The results we analyzed from the analysis of deflection angle $\Theta$ by Kerr-Newman BH with quintessence are generalized as follows:

Deflection angle by Kerr-Newman BH with quintessence with respect to Impact parameter:

- The behavior of $\Theta$ w.r.t $b$ can be analyzed for the particular range of $b$.

- We can analyzed the deflection angle by Kerr-Newman BH with quintessence for $0.01 \leq \alpha \leq 1$.

- We observe that for the variation of $Q$ the deflection angle goes from negative to positive and then decreasing which shows that the deflection angle initially at non-physical unstable state and then goes to stable stable.

- For positive values of $a$, the deflection angle exponentially decreases then become identical. We can not observe any stable behavior for $a<1$.

\section{Acknowledgement}

This work is supported by Comisión Nacional de Ciencias y Tecnología of Chile (CONICYT) through FONDECYT Grant No 3170035 (A. Ö.).

[1] A. Einstein, “The Foundation of the General Theory of Relativity," Annalen Phys. 49, no. 7, 769 (1916) [Annalen Phys. 14, 517 (2005)].

[2] W. de Sitter, “Einstein's theory of gravitation and its astronomical consequences, Third Paper," Mon. Not. Roy. Astron. Soc. 78, 3 (1917).

[3] M. Barriola and A. Vilenkin, “Gravitational Field of a Global Monopole,” Phys. Rev. Lett. 63, 341 (1989).

[4] P. Jetzer, "Boson stars," Phys. Rept. 220, 163 (1992).

[5] T. D. Lee and Y. Pang, “Nontopological solitons," Phys. Rept. 221, 251 (1992).

[6] R. D. Viollier, F. R. Leimgruber and D. Trautmann, “Halos of heavy neutrinos around baryonic stars," Phys. Lett. B 297, 132 (1992).

[7] N. Bilic and R. D. Viollier, “Gravitational phase transition of heavy neutrino matter,” Phys. Lett. B 408, 75 (1997).

[8] K. Akiyama et al. [Event Horizon Telescope Collaboration], "First M87 Event Horizon Telescope Results. I. The Shadow of the Supermassive Black Hole," Astrophys. J. 875, no. 1, L1 (2019).

[9] S. S. Zhao and Y. Xie, "Strong deflection gravitational lensing by a modified Hayward black hole," Eur. Phys. J. C 77, no. 5, 272 (2017).

[10] R. A. Konoplya, "Shadow of a black hole surrounded by dark matter," Phys. Lett. B 795, 1 (2019).

[11] C. Bambi, K. Freese, S. Vagnozzi and L. Visinelli, "Testing the rotational nature of the supermassive object M87* from the circularity and size of its first image," arXiv:1904.12983 [gr-qc].

[12] R. Shaikh, "Black hole shadow in a general rotating spacetime obtained through Newman-Janis algorithm," arXiv:1904.08322 [gr-qc].

[13] A. B. Abdikamalov, A. A. Abdujabbarov, D. Ayzenberg, D. Malafarina, C. Bambi and B. Ahmedov, "A black hole mimicker hiding in the shadow: Optical properties of the $\gamma$ metric," arXiv:1904.06207 [gr-qc].

[14] A. Abdujabbarov, B. Ahmedov, N. Dadhich and F. Atamurotov, “Optical properties of a braneworld black hole: Gravitational lensing and retrolensing," Phys. Rev. D 96, no. 8, 084017 (2017). 
[15] A. Abdujabbarov, B. Juraev, B. Ahmedov and Z. Stuchlk, "Shadow of rotating wormhole in plasma environment," Astrophys. Space Sci. 361, no. 7, 226 (2016).

[16] A. Abdujabbarov, M. Amir, B. Ahmedov and S. G. Ghosh, “Shadow of rotating regular black holes,” Phys. Rev. D 93, no. 10, 104004 (2016).

[17] A. Abdujabbarov, B. Toshmatov, Z. Stuchlk and B. Ahmedov, "Shadow of the rotating black hole with quintessential energy in the presence of plasma," Int. J. Mod. Phys. D 26, no. 06, 1750051 (2016).

[18] U. Papnoi, F. Atamurotov, S. G. Ghosh and B. Ahmedov, "Shadow of five-dimensional rotating Myers-Perry black hole," Phys. Rev. D 90, no. 2, 024073 (2014).

[19] F. Atamurotov, A. Abdujabbarov and B. Ahmedov, "Shadow of rotating non-Kerr black hole," Phys. Rev. D 88, no. 6, 064004 (2013).

[20] E. J. Copeland, M. Sami and S. Tsujikawa, “Dynamics of dark energy," Int. J. Mod. Phys. D 15, 1753 (2006).

[21] P. J. E. Peebles and B. Ratra, "The Cosmological constant and dark energy," Rev. Mod. Phys. 75, 559 (2003).

[22] A. G. Riess et al. [Supernova Search Team], "Type Ia supernova discoveries at $\mathrm{z}>1$ from the Hubble Space Telescope: Evidence for past deceleration and constraints on dark energy evolution," Astrophys. J. 607, 665 (2004).

[23] F. Kottler, “Uber die physikalischen Grundlagen der Einsteinschen Gravitationstheorie," Ann. Phys. (Berlin) 361, (1918)401.

[24] http://www.thphys.uni-heidelberg.de/ wetterich/DEBarcelona0706.

[25] G. W. Gibbons and M. C. Werner, "Applications of the Gauss-Bonnet theorem to gravitational lensing," Class. Quant. Grav. 25, 235009 (2008)

[26] M. C. Werner, "Gravitational lensing in the Kerr-Randers optical geometry," Gen. Rel. Grav. 44,3047 (2012)

[27] A. Ishihara, Y. Suzuki, T. Ono, T. Kitamura and H. Asada, "Gravitational bending angle of light for finite distance and the Gauss-Bonnet theorem," Phys. Rev. D 94, no. 8, 084015 (2016).

[28] H. Arakida, "Light deflection and GaussBonnet theorem: definition of total deflection angle and its applications," Gen. Rel. Grav. 50, no. 5, 48 (2018).

[29] T. Ono, A. Ishihara and H. Asada, "Deflection angle of light for an observer and source at finite distance from a rotating wormhole," Phys. Rev. D 98, no. 4, 044047 (2018).

[30] T. Ono, A. Ishihara and H. Asada, "Gravitomagnetic bending angle of light with finite-distance corrections in stationary axisymmetric spacetimes," Phys. Rev. D 96, no. 10, 104037 (2017).

[31] K. Jusufi, A. Övgün and A. Banerjee, "Light deflection by charged wormholes in Einstein-Maxwell-dilaton theory," Phys. Rev. D 96, no. 8, 084036 (2017) Addendum: [Phys. Rev. D 96, no. 8, 089904 (2017)].

[32] A. Övgün, K. Jusufi and I. Sakalli, “Exact traversable wormhole solution in bumblebee gravity,” Phys. Rev. D 99, no. 2, 024042 (2019).

[33] K. Jusufi and A. Övgün, “Gravitational Lensing by Rotating Wormholes,” Phys. Rev. D 97, no. 2, 024042 (2018).

[34] K. Jusufi and A. Övgün, "Light Deflection by a Quantum Improved Kerr Black Hole Pierced by a Cosmic String," doi:10.1142/S0219887819501160 arXiv:1707.02824 [gr-qc].

[35] K. Jusufi, M. C. Werner, A. Banerjee and A. Övgün, "Light Deflection by a Rotating Global Monopole Spacetime," Phys. Rev. D 95, no. 10, 104012 (2017).

[36] K. Jusufi, I. Sakalli and A. Övgün, “Effect of Lorentz Symmetry Breaking on the Deflection of Light in a Cosmic String Spacetime," Phys. Rev. D 96, no. 2, 024040 (2017).

[37] T. Ono, A. Ishihara and H. Asada, "Deflection angle of light for an observer and source at finite distance from a rotating global monopole," arXiv:1811.01739 [gr-qc].

[38] A. Övgün, "Weak gravitational lensing of regular black holes with cosmic strings using the Gauss-Bonnet theorem," Phys. Rev. D 99, no. 10, 104075 (2019).

[39] G. Crisnejo and E. Gallo, "Weak lensing in a plasma medium and gravitational deflection of massive particles using the Gauss-Bonnet theorem. A unified treatment," Phys. Rev. D 97, no. 12, 124016 (2018)

[40] I. Sakalli and A. Ovgun, "Hawking Radiation and Deflection of Light from Rindler Modified Schwarzschild Black Hole," EPL 118, no. 6, 60006 (2017).

[41] A. Övgün, G. Gyulchev and K. Jusufi, “Weak Gravitational lensing by phantom black holes and phantom wormholes using the Gauss-Bonnet theorem," Annals Phys. 406, 152 (2019).

[42] K. Jusufi and A. Övgün, "Effect of the cosmological constant on the deflection angle by a rotating cosmic string," Phys. Rev. D 97, no. 6, 064030 (2018).

[43] K. Jusufi, A. Övgün, J. Saavedra, Y. Vasquez and P. A. Gonzalez, “Deflection of light by rotating regular black holes using the Gauss-Bonnet theorem," Phys. Rev. D 97, no. 12, 124024 (2018).

[44] A. Övgün, "Light deflection by Damour-Solodukhin wormholes and Gauss-Bonnet theorem," Phys. Rev. D 98, no. 4, 044033 (2018).

[45] A. Övgün, K. Jusufi and I. Sakalli, “Gravitational lensing under the effect of Weyl and bumblebee gravities: Applications of GaussBonnet theorem," Annals Phys. 399, 193 (2018).

[46] A. Övgün, "Deflection angle of photon through dark matter by black holes and wormholes using the Gauss-Bonnet theorem," Universe 5, 115 (2019)

[47] A. Övgün, I. Sakalli and J. Saavedra, "Weak gravitational lensing by Kerr-MOG Black Hole and Gauss-Bonnet theorem," arXiv:1806.06453 [gr-qc].

[48] A. Övgün, I. Sakalli and J. Saavedra, "Shadow cast and Deflection angle of Kerr-Newman-Kasuya spacetime," JCAP 1810, no. 10, 041 (2018).

[49] W. Javed, R. Babar and A. Övgün, "The effect of the Brane-Dicke coupling parameter on weak gravitational lensing by wormholes and naked singularities," Phys. Rev. D 99, no. 8, 084012 (2019) 
[50] W. Javed, R. Babar and A. Övgün, "Effect of the Dilaton Field on Deflection Angle of Massive Photons by Black Holes in Einstein-Maxwell-Dilaton-Axion," Theory. Preprints 2019, 2019050148 (doi: 10.20944/preprints201905.0148.v1)

[51] W. Javed, J. Abbas, and A. Övgün, " Deflection Angle of Photon from Magnetized Black Hole and Effect of Nonlinear Electrodynamics," Preprints 2019, 2019030260 (doi: 10.20944/preprints201903.0260.v1)

[52] Z. Xu and J. Wang, “Kerr-Newman-AdS Black Hole In Quintessential Dark Energy,” Phys. Rev. D 95, no. 6, 064015 (2017).

[53] Chandrasekhar S.: The Mathematical Theory of Black Holes. Oxford University Press, New York (1983).

[54] C. Lammerzahl, M. Maceda and A. Macas, "On slowly rotating black holes and nonlinear electrodynamics," Class. Quant. Grav. 36, no. 1, 015001 (2019).

[55] M. Visser, “The Kerr spacetime: A Brief introduction," arXiv:0706.0622 [gr-qc].

[56] W. Javed and R. Babar, "Fermions Tunneling and Quantum Corrections for Quintessential Kerr-Newman-AdS Black Hole," Adv. High Energy Phys. 2019, 2759641 (2019).

[57] Z. Xu and J. Wang, “Kerr-Newman-AdS Black Hole In Quintessential Dark Energy," Phys. Rev. D 95, no. 6, 064015 (2017). 